\title{
Experimental study of confined coaxial jets in a non-axisymmetric co-flow
}

\author{
I. A. Sofia Larsson ${ }^{1}$ [ $\cdot$ Henrik Lycksam ${ }^{1} \cdot$ T. Staffan Lundström ${ }^{1} \cdot$ B. Daniel Marjavaara ${ }^{2}$
}

Received: 23 April 2020 / Revised: 28 October 2020 / Accepted: 30 October 2020 / Published online: 20 November 2020

(c) The Author(s) 2020

\begin{abstract}
Confined, turbulent, coaxial jets in a non-axisymmetric co-flow are studied using particle image velocimetry (PIV) and planar laser-induced fluorescence (PLIF) simultaneously. Eight different cases are measured. Two momentum flow ratios of the co-flow are used in the experiment to investigate the effect on the coaxial burner jet behavior and mixing characteristics of the coaxial jet flow and the co-flowing, secondary fluid. In addition, four different momentum flow ratios of the coaxial outer to inner jet are investigated. The objective of the study is to get a deeper understanding of how the flow dynamics affects the entrainment and mixing process in a coaxial jet with a non-axisymmetric, surrounding co-flow. The results show that the introduction of a coaxial stream affects the inner jet and decreases the mixing with the surrounding co-flow; the effect is enhanced as the momentum flow ratio of the coaxial jet increases. The distribution of the secondary, co-flowing fluid controls the shape and direction of the coaxial jet, but does not have a significant impact on the mixing process near the centerline. Practical implications of this investigation are related to the possibility to better control a diffusion flame by introducing a coaxial stream. In this context it is concluded that it is possible to affect the jet development and hence the flame length. The conclusion is based on the assumption that the outer, coaxial stream has a low mass flow, not enough to provide complete combustion, and hence the co-flowing, secondary fluid provides the air needed for the combustion process.
\end{abstract}

I. A. Sofia Larsson

sofia.larsson@1tu.se

1 Division of Fluid and Experimental Mechanics, Luleå

University of Technology, SE-97187 Luleå, Sweden

2 LKAB, SE-98186 Kiruna, Sweden 


\section{Graphic abstract}
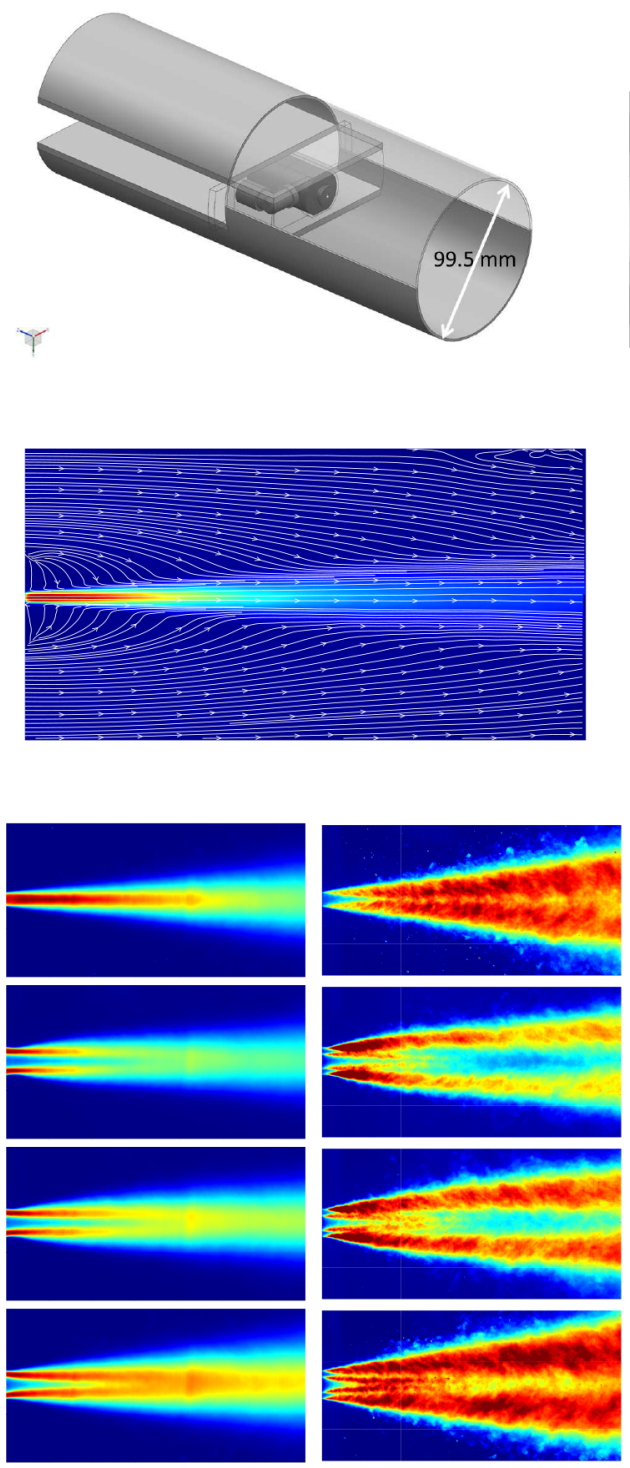
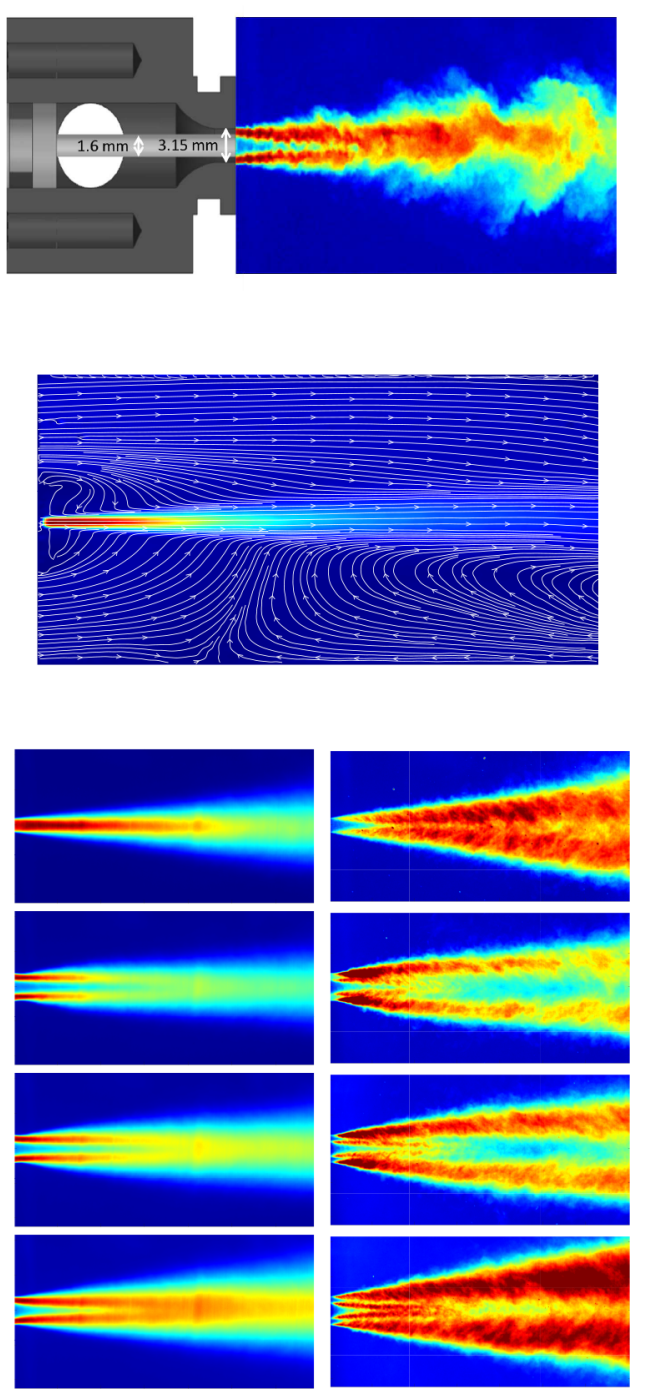

\section{Introduction}

Confined, coaxial jets consist of an inner jet with a surrounding coaxial flow, issued into a still or co-flowing environment. Their mixing capabilities make them important in many different applications, including burners and, especially, diffusion flames where the fuel and air enter separately as turbulent jets. This means that the necessary combustion air needs to be entrained and mixed with the fuel. The length and shape of the flame largely depend on the mixing process between the fuel and the air. The flow characteristics directly affect the mixing rate which is the slowest step of the combustion process affecting the efficiency of the combustion. Hence, by studying turbulent, coaxial jets, important predictions regarding flame characteristics and the effect of different flow parameters on the combustion process can be made (Lotfiani et al. 2013).

Confined, coaxial jets occur in a number of engineering devices. Compared to free jets, a confined jet has an axial pressure gradient and the possibility of reversed flow. The jet develops in a surrounding coaxial stream and in an imaginary constant-area duct the pressure gradient in the main flow direction is a result of the momentum transfer from the primary to the entrained secondary stream. The total mass flow rate in the duct remains constant while the momentum flux varies with axial distance consistent with 
the changes in pressure. The pressure gradient influences the spreading and entrainment rates and the relative strengths of the coaxial streams, and the dimensions of the nozzles and mixing duct determine the pressure variation and the flow pattern (Gibson 1986).

Despite the relatively simple geometry of a coaxial jet (see Fig. 1), there are several parameters defining the configuration that can influence the flow development. These parameters include the inner and outer velocities $\left(U_{\mathrm{i}}\right.$ and $U_{\mathrm{o}}$, respectively), the geometry of the exit (mainly defined by the inner and outer diameter $D_{\mathrm{i}}$ and $D_{\mathrm{o}}$ as well as by the shape and thickness of the separating wall between the two streams), the characteristics of the exit boundary layers (thicknesses and state), and the freestream turbulence. A complete understanding of the flow field and behavior is still missing, which is easily understood by the large amounts of parameters involved (Segalini and Talamelli 2011). A very important parameter for coaxial jets is the momentum ratio which, if the densities of the two streams are equal, can be reduced to the velocity ratio, $r_{u}$, according to

$M=\frac{\rho_{\mathrm{o}} U_{\mathrm{o}}^{2}}{\rho_{\mathrm{i}} U_{\mathrm{i}}^{2}} \longrightarrow\left(\rho_{\mathrm{i}}=\rho_{\mathrm{o}}\right) \longrightarrow r_{\mathrm{u}}=\frac{U_{\mathrm{o}}}{U_{\mathrm{i}}}$

For Reynolds number high enough, $>10^{3}-10^{4}$, the momentum ratio is the main parameter that influences the flow and determines its evolution.

The initial jet spreading rate and the length of the initial merging region are sensitive to the nozzle geometry including the inner duct and the inlet flow conditions (Ahmed and Sharma 2000). The overall coaxial jet dynamics and its vortex topology are also strongly dependent on the shape of the inlet nozzle. Different shapes lead to significant variations of the shear layers that are present in coaxial jets (Balarac and Métais 2005). An important parameter of the nozzle geometry is the ratio of outer to inner nozzle diameters $D_{\mathrm{o}} / D_{\mathrm{i}}$, and it has been observed that an increase of this value causes an increase in the inner potential core length (Rehab et al. 1997; da Silva et al. 2003).

In the near field region close to the nozzle, the vortex pattern strongly depends on $r_{\mathrm{u}}$ as well as the Reynolds number of both streams. There is a complex interaction between the vortices of the inner and outer shear layers that affects the

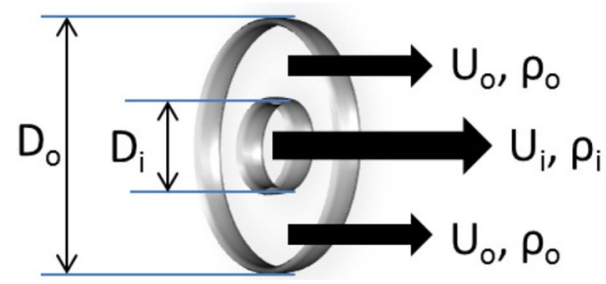

Fig. 1 Schematic of a coaxial jet instability mechanisms in the near field region (Dahm and Tryggvason 1992). The initial growth of the shear layers is controlled by Kelvin-Helmholtz instabilities giving rise to the development of regular vortices within the first diameters of the jet (Favre-Marinet et al. 1999). An interesting observation is that keeping the velocity ratio constant while varying the absolute velocities, i.e. the Reynolds number of both streams, results in a dramatically different flow topology, due to the difference in the initial shear layer momentum thicknesses. To exemplify, decreasing the initial vorticity thickness may lead to a faster roll-up process of vortices in the shear layers and large effects on the downstream flow (Rehab et al. 1997; da Silva et al. 2003).

As stated above, by varying the velocity ratio $r_{\mathrm{u}}=U_{\mathrm{o}} / U_{\mathrm{i}}$, it is possible to significantly change the flow dynamics. For small $r_{u}$, the inner jet determines the main instability in the inner shear layer while the outer shear layer develops its own preferred instability separately (Segalini and Talamelli 2011). When $r_{\mathrm{u}}<1$ and $U_{\mathrm{o}}$ are also relatively small, the outer stream acts mainly as a co-flowing velocity which does not substantially modify the inner jet dynamics. For $r_{\mathrm{u}}<0.1$, the outer flow has only a minor influence on the spatial jet evolution (da Silva et al. 2003).

For large $r_{\mathrm{u}}$, there is a clear dominance of the outer shear layer that forces the inner one to follow its dynamics (da Silva et al. 2003). Coaxial jets operating with a large $r_{\mathrm{u}}$ are used for its ability to destabilize, fragment and mix the central stream in the outer, rapid stream (Villermaux and Rehab 2000).

It is well known that small-scale mixing is mainly governed by the small-scale turbulence level, whereas largescale mixing is controlled by the large-scale coherent structures. It is therefore very important to understand the detailed dynamics of these structures (da Silva et al. 2003). The near field of a coaxial jet is an example of a relatively complex mixing process (Schumaker and Driscoll 2012).

Entrainment plays a crucial role in the mixing process and occurs due to vortices. The first step in the mixing process is kinematic, involving large-scale vortical structures entraining ambient fluid into the jet as they undergo pairing. Turbulent, and finally, molecular diffusion then completes the mixing process at the smallest scales. It is clear that the key step in the mixing process is the rate at which ambient fluid is entrained (Sreenivas 2000). In reactive cases, the entrainment affects the residence time through the flame and the overall chemical reaction. Therefore, studying the flow field dynamics and the entrainment of the jet is of fundamental interest (Han and Mungal 2001).

The velocity ratio has a very strong influence on the turbulent mixing process. The rate of momentum transfer between the jets increases as $r_{\mathrm{u}}$ is increased (>1), promoting faster mixing. Measurements show that density effects on mixing are rather well taken into account by considering 
the momentum ratio, instead of $r_{\mathrm{u}}$ and the density ratio separately. This observation confirms the important role played by the momentum ratio in the dynamics of variable density coaxial jets (Favre-Marinet and Schettini 2001).

In this work, the focus is on the diffusion flame in a rotary kiln, which is a cylindrical-shaped, long, rotating oven with a burner at one end, and it is used in various industrial applications to heat up materials to high temperatures. The kiln of interest here is used in the iron ore pelletizing process to sinter the pellets to increase their strength. A down-scaled, isothermal model consisting of a confined, coaxial jet issuing into a co-flowing surrounding is studied. There are two features that distinguish this investigation from most of the studies presented in the literature: (1) coaxial jets in a coflow are examined and (2) this co-flow is non-axisymmetric. This results in a very complex, instationary flow field with several interesting flow phenomena. Figure 2 shows a schematic of the kiln inlet geometry where $\dot{m}_{1}$ and $\dot{m}_{2}$ represent the mass flow through the upper and lower co-flow inlet, respectively.

In a reactive flow, mixing and reaction interact with each other, making understanding of mixing more challenging. It is therefore desirable to study a mixing process with similar characteristics to a reactive flow, but without the influence of the reaction (Li et al. 2017). To be able to do this, a number of theories for confined jets have been developed, making it possible to describe and define the properties and mixing characteristics of an enclosed diffusion flame. With the aid of these theories, similarity or scaling parameters can be found which can be used to scale experimental isothermal models of reacting flows in general and the mixing in particular. The scaling parameter must be preserved between the real plant and the model, and this is obtained by correcting for the variable densities in combusting systems by distorting the nozzle diameter relative to the duct diameter or the nozzle flow. This ensures that the momentum ratio of the co-flow relative to the jet in the model matches that of the reacting system (Mullinger and Jenkins 2008; Parham

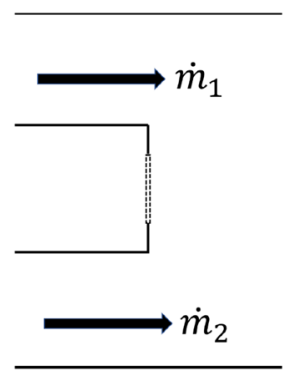

(a) Side view

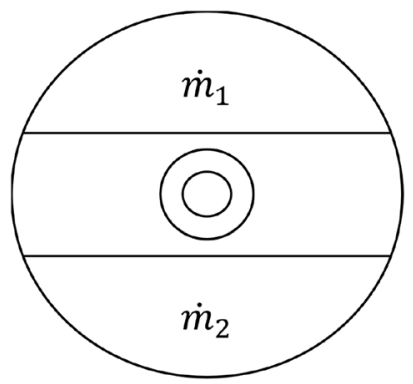

(b) Front view
Fig. 2 Schematic of the kiln inlet geometry; the size of the coaxial jet nozzle is exaggerated for better clarity et al. 2005). In this work, the Craya-Curtet parameter was deemed the most suitable scaling parameter (Larsson et al. 2015a). It is essentially the momentum ratio of the jets (the nozzle flow and surrounding co-flow) and a measure of the dynamic mixing. It is always positive and varies between zero and infinity. Depending on the value of the parameter, the flame has different characteristics. In this work, the Craya-Curtet parameter equals 1.08 for a single jet with no outer coaxial stream, indicating a long flame. The value has been calculated based on the process parameters and is used for scaling the model to ensure that the results acquired are indicative also for the real process. The introduction of the outer coaxial stream implies that the Craya-Curtet parameter increases, changing the jet characteristics. How this affects the mixing process of the flow supplied through the coaxial nozzle with the co-flow is the main interest of this study.

Experiments using particle image velocimetry (PIV) and planar laser-induced fluorescence (PLIF) simultaneously are performed. In this way, both the flow field and the resulting mixing of a passive scalar can be studied. The objective of the study is to get a deeper understanding of how the flow dynamics affects the entrainment and mixing process in a coaxial jet with a non-axisymmetric surrounding co-flow.

\section{Method}

The coaxial jet system is a simplified, downscaled model of the kiln of interest. It is built in PMMA and FEP (transparent plastics) to enable optical, laser-based measurements using PIV and PLIF. The geometry consists of a cylinder with inner diameter $D=100 \mathrm{~mm}$ with two so-called secondary flow inlets approximated as semicircles separated by a wall, called back plate. The back plate is centered with respect to $y / D=0$ and its height is $D / 3$ and in the middle the coaxial jet nozzle is located. This nozzle is built in metal and consists of an inner jet with $D_{\mathrm{i}}=1.6 \mathrm{~mm}$ and a coaxial, annular jet surrounding the inner one with $D_{\mathrm{o}}=3.15 \mathrm{~mm}$ yielding a diameter ratio $\approx 2$. As mentioned in the previous section, the inner jet of the nozzle has been scaled using the Craya-Curtet parameter. A detailed drawing of the geometry of the test section and the coaxial jet nozzle can be seen in Fig. 3.

The chosen fluid for the physical model is water, allowing the smaller scale of the model kiln. Three different pumps are used, one for the secondary flow and two for the coaxial jet. The flow through each secondary flow inlet is controlled by manual valves and monitored with magnetic flow meters (Krohne Optiflux DN50) to an accuracy of reading of $0.1 \%$. The pumps feeding the coaxial jet is electrically controlled and monitored with magnetic flow meters (ABB Hygienicmaster FEH311) to an accuracy of reading of $0.4 \%$. The 

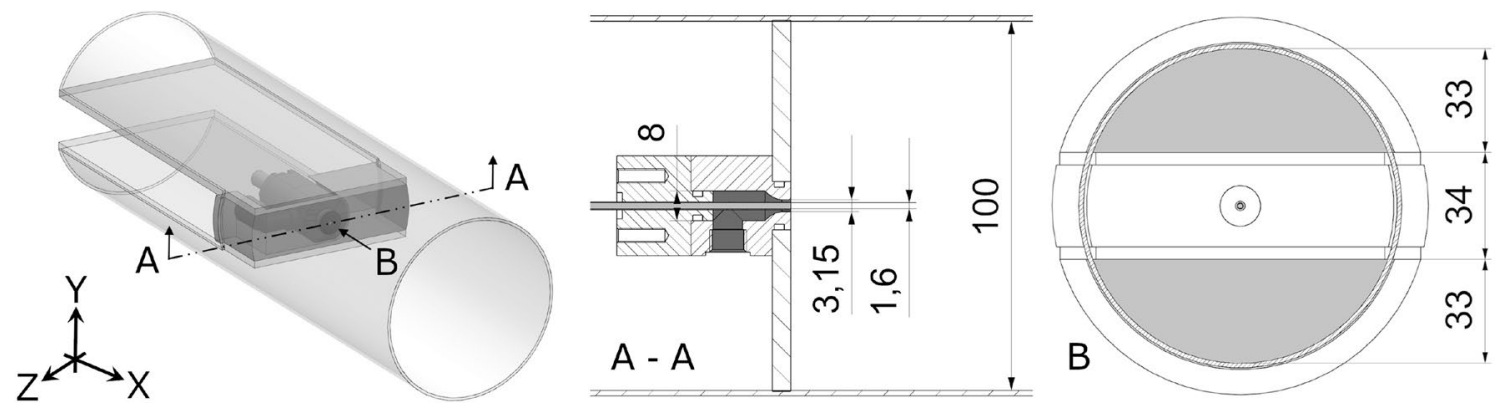

Fig. 3 Kiln geometry with a cut-through of the coaxial nozzle with dimensions in $\mathrm{mm}$. The secondary, co-flowing fluid is supplied through the semicircular openings (shaded in gray in section B) above and below the back plate with the coaxial nozzle in the middle

temperature of the working fluid in the setup is controlled to $22{ }^{\circ} \mathrm{C} \pm 0.3{ }^{\circ} \mathrm{C}$ using a cooling system in the tank.

\subsection{Measuring system}

The measuring system used is a commercially available system from Lavision $\mathrm{GmbH}$. It consists of a double-pulsed Nd-YAG laser from Litron $(532 \mathrm{~nm}, 15 \mathrm{~Hz}, 200 \mathrm{~mJ})$ with a laser-guiding arm, two sCMOS cameras (5.5 MP, 16 bit, $6.5 \times 6.5 \mu \mathrm{m}$ pixel size), a programmable timing unit (PTU $\mathrm{X})$ for triggering and synchronization, and a computer with the software Davis 8.4 to control the system and store the data. For the PIV/PLIF measurements, two cameras were used mounted on top of each other, and sheet optics generated a $2 \mathrm{~mm}$-thick laser sheet and focused it on the desired location (with an accuracy of $0.5 \mathrm{~mm}$ ). Each camera was mounted with $100 \mathrm{~mm} \mathrm{f} / 2.8$ macro lenses, and the camera recording the PIV images was fitted with a bandpass filter, while the camera recording the PLIF images had a cutoff filter. Both the PIV and PLIF camera were mounted with a slight angle $\left(\approx 5^{\circ}\right)$ with respect to a line normal to the laser sheet. To enable comparisons of the measurement results in the same plane and determine the scale factor from pixel to $\mathrm{mm}$, a calibration was made using a target with known spacing which was inserted through the outlet and placed in the area of interest. A pinhole model was applied to correct for the optical distortion and the final calibration error was below 0.7 pixel. Figure 4 shows an illustration of the experimental setup.

The tracer particles used for the PLIF measurements were fluorescent dye rhodamine $590 \mathrm{Cl}$, while the PIV particles used were hollow glass spheres, HGS-10, with a diameter of $10 \mu \mathrm{m}$ from Dantec Dynamics.

\subsection{Data acquisition}

The Reynolds number, Re, in the real full-size kiln is approximately $3.5 \times 10^{5}$, based on its diameter. In the experiments, a reduced $\mathrm{Re}$ is used due to the limitation of the

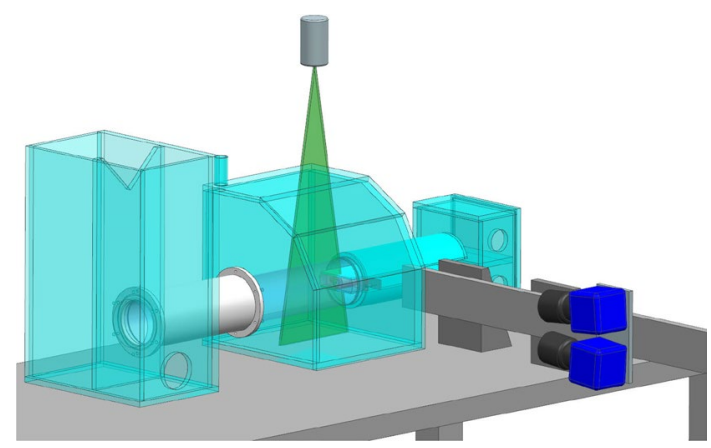

Fig. 4 Experimental setup

pumps feeding the coaxial jet nozzle. This is not an issue though, since $\mathrm{Re}$ is still in the fully turbulent range and reduced Reynolds similarity holds for turbulent jets (Pope 2000). The coaxial jet has an Re varying between 11,000 and 23,000, based on the mass weighted jet velocity and the geometric equivalent nozzle diameter, depending on the flow case studied. For a single jet (with no outer coaxial flow), Re is based on the jet velocity and the inner diameter $D_{\mathrm{i}}$. The surrounding co-flow of secondary fluid has a Reynolds number of 10000 based on the total mass flow through the two inlets and the kiln diameter, $D$.

Two momentum flow ratios, $M_{\mathrm{sec}}$, based on the secondary flow through each separate inlet are used in the experiment to investigate the effect on the coaxial burner jet behavior and mixing characteristics of the coaxial jet flow and the secondary fluid. To facilitate the comparison between the cases, the momentum flow in each secondary inlet is approximated with the mass flow squared, $\dot{m}_{1}$ for the upper inlet and $\dot{m}_{2}$ for the lower inlet, $M_{\mathrm{sec}} \approx\left(\dot{m}_{1} / \dot{m}_{2}\right)^{2}$. For reference, see Fig. 2 . This is a simplification, but the only purpose is to describe the different mass flow distributions between the secondary fluid inlets in a convenient way. A momentum flow ratio of one corresponds to matched mass flow between the inlets, and increasing the ratio means that the mass flow distribution becomes uneven with a dominating upper flow. The 
total mass flow through both secondary inlets is kept constant throughout the measurements.

In addition, four different momentum flow ratios $\left(M_{\text {jet }}=\left(\rho_{\mathrm{o}} U_{\mathrm{o}}^{2} A_{\mathrm{o}}\right) /\left(\rho_{\mathrm{i}} U_{\mathrm{i}}^{2} A_{\mathrm{i}}\right)\right)$ of the coaxial outer to inner jet are investigated. In total there are therefore eight different cases measured and Table 1 summarizes all the flow parameters in the experiments and provides an overview of the cases studied. $M_{\text {jet }}=0$ corresponds to a single jet. The highest momentum ratio corresponds to the maximum capacity of the outer coaxial pump. The total mass flow through the coaxial jet nozzle depends on the flow case studied. The inner jet is always kept at a constant mass flow, while the mass flow through the outer jet is varied to obtain the wanted momentum flow ratio. This approach therefore changes the value of the Craya-Curtet parameter and consequently the jet characteristics, which is the main interest of this work.

Images are taken with a frequency of $15 \mathrm{~Hz}$ during $10 \mathrm{~s}$, corresponding to a total of 150 image pairs for each recorded set (ensuring a statistically converged mean).

Due to the large velocity differences between the coaxial burner jets and the secondary fluid, two recordings were made with different time interval, $\mathrm{d} t$, between the laser pulses. This procedure was applied in an attempt to resolve the different flow regions originating from the wide velocity range. The $\mathrm{d} t$ was varied between 25 and $3000 \mu \mathrm{s}$.

To be able to see the mixing between all different streams (inner and outer coaxial jet and co-flowing, secondary fluid), the rhodamine was injected through the outer coaxial jet nozzle. When this flow was zero, the rhodamine was injected through the inner coaxial jet instead.

\subsection{Data processing}

The acquired images were processed in two different ways depending on the measuring method. For the PIV, first a

Table 1 Flow cases studied

\begin{tabular}{lll}
\hline & Mom. flow ratio, $M_{\text {jet }}$ & $\begin{array}{l}\text { Veloc- } \\
\text { ity ratio, } \\
u_{\mathrm{o}} / u_{\mathrm{i}}\end{array}$ \\
\hline $\begin{array}{l}\text { Sec. flow distribution, } \\
M_{\text {sec }}=1\end{array}$ & 0 & 0 \\
& 0.5 & 0.48 \\
& 1 & 0.69 \\
& 3.8 & 1.33 \\
Sec. flow distribution, & 0 & 0 \\
$M_{\text {sec }}=5.43$ & 0.5 & 0.48 \\
& 1 & 0.69 \\
& 3.8 & 1.33 \\
\hline
\end{tabular}

For each $M_{\text {sec }}$, four different $M_{\text {jet }}$ (with corresponding velocity ratio $\left.u_{\mathrm{o}} / u_{\mathrm{i}}\right)$ are studied background subtraction and a min/max filter for particle intensity normalization were applied, followed by a multipass scheme with decreasing window size and offset to calculate the particle displacement. The interrogation window size was $64 \times 64$ pixels for the first pass and $32 \times 32$ pixels for the second and third passes with adaptive window shift, both with an overlap of $75 \%$. The cross-correlation was performed using the standard cyclic FFT algorithm with a three-point Gaussian peak fit to estimate the sub-pixel displacement, followed by vector post-processing by applying a median filter to reject spurious vectors and to interpolate from surrounding interrogation windows.

Since the PLIF recording was done simultaneously with the PIV recording, 150 image pairs were also captured with the PLIF camera. The first frame of each pair was chosen for the PLIF analysis. The mean background image was subtracted to remove background noise, and to account for the non-uniformity of the laser sheet, intensity correction was applied to the raw PLIF image.

Since it is not possible to keep the rhodamine concentration constant between the cases, the PLIF images need to be scaled to be able to compare the results.

An area, close to the nozzle where the jet still contains a large amount of pure jet fluid, is chosen for calculation of a mean, maximum value that is used as reference. The intensity in all pixels over the entire image is then divided by this reference intensity. Since the reference value is a mean, it does not correspond to the exact maximum intensity in the picture and hence the scale ranges from 0 to a value slightly higher than 1, where 1 should correspond to "pure" burner jet fluid. Since the absolute concentration of rhodamine in the water was not of interest, only the comparison of different cases, this procedure of processing the data is justified. The PLIF images are cropped a distance from the upper and lower wall to highlight the jet.

It should be noted that since the laser sheet has a Gaussian profile and a slightly larger thickness than the inner diameter of the coaxial jet nozzle, a part of the outer coaxial jet is also illuminated closest to the nozzle. As a result, the central part of the coaxial jet shows a concentration that deviates slightly from zero closest to the nozzle where the jet has not yet started spreading. The problem with achieving perfect alignment of the laser sheet with the centerline of the kiln also adds to this deviation. It is however not an issue for the analysis of the measurement data.

The major error sources in a PLIF experiment are mainly connected to the calibration process and non-uniformity of the laser sheet with temporal and spatial energy fluctuations (Melton and Lipp 2003). Since no calibration was performed to determine the relationship between rhodamine concentration and light intensity, the focus was instead on the errors connected to the PIV measurements and the resulting velocity vectors. The radial velocity is the weakest component 
and hence the hardest one to measure accurately. Since the focus in this work lies on the axial velocity, the uncertainty reported concerns the axial component only. The experimental uncertainties regarding pixel displacement and hence velocity were calculated directly by the software (Wieneke 2015 ) revealing a result of maximum $4 \%$. The highest uncertainties can be found in the first few nozzle diameters of the flow. In addition to this, three replicate measurements of six different flow cases were performed to be able to do a repeatability test. Following Coleman and Steele (1999) yields a maximum precision error of about $8 \%$. It should however be noted that this high value corresponds to a region with a sudden increase in velocity. The mean precision error over the entire field of view is well below $2 \%$. A careful setup of the experiment based on previous experience ensures a minimized systematic error. The random errors are therefore most likely to dominate over the systematic errors, and the total uncertainty in the measurement of the velocity is therefore about $4 \%$.

\section{Results and discussion}

To start with, the main flow features will be exposed followed by a presentation of axial profiles. Then focus is set on centerline values, Reynolds fluxes, instantaneous images, and spreading rates and finally the mixing is quantified.

\subsection{Flow overview}

The flow field in a kiln similar to the present one has been studied extensively, with and without a burner jet in the middle of the geometry (Larsson et al. 2012a, b, 2015a, b, c, 2016), both numerically and experimentally. The general flow features can be seen in Fig.5.

The back plate separating the two secondary inlets acts like a bluff body to the flow, resulting in vortices shedding from the edges and a wake developing behind it. When the burner jet is not present in the setup, a recirculation zone occurs in this wake region. Its extension depends on the direction of the incoming secondary flow. When the burner jet is included and the mass flow distribution between the secondary inlets is uneven with a dominating flow from the upper inlet (Fig. 5b), a recirculation zone in the lower part of the kiln develops. This is a result of the entrainment of fluid emerging from the lower secondary inlet into the primary burner jet.

To get an overview of the flow field, contour plots showing the time mean and root mean square (rms) fluctuations of concentration are shown for each case in Fig. 6 . The jet development and spreading can be seen as well as the shear layers emanating from the velocity gradients between the different streams. The PIV results require an optimized time between exposures, $\mathrm{d} t$, to capture the jet accurately, and the correlation results in an unavoidable spatial averaging. Therefore, the concentration field can provide a more detailed overview compared to the velocity field. However, they are connected and by studying the concentration field and particularly the concentration fluctuations, it is possible to draw conclusions about the convective mass transfer and the impact of turbulence on mixing. The area closest to the nozzle is the most interesting part from a combustion perspective since, as thoroughly described by Villermaux and Rehab (2000), "when two fluid streams liable to react chemically merge, the extent and topology of the reaction zone coincide, provided the chemical reaction has very fast kinetics, with the region of diffusive interpenetration between the streams." Hence, the focus is on this area.

Figure 6 reveals a distinct difference in shear layer evolution between a single jet, with no outer coaxial flow, and a coaxial jet. A single jet has shear layers between the jet and surrounding secondary fluid, while a coaxial jet has shear layers both between the inner and outer coaxial jet as well as between the outer coaxial jet and the surrounding co-flowing, secondary fluid. This can clearly be seen when
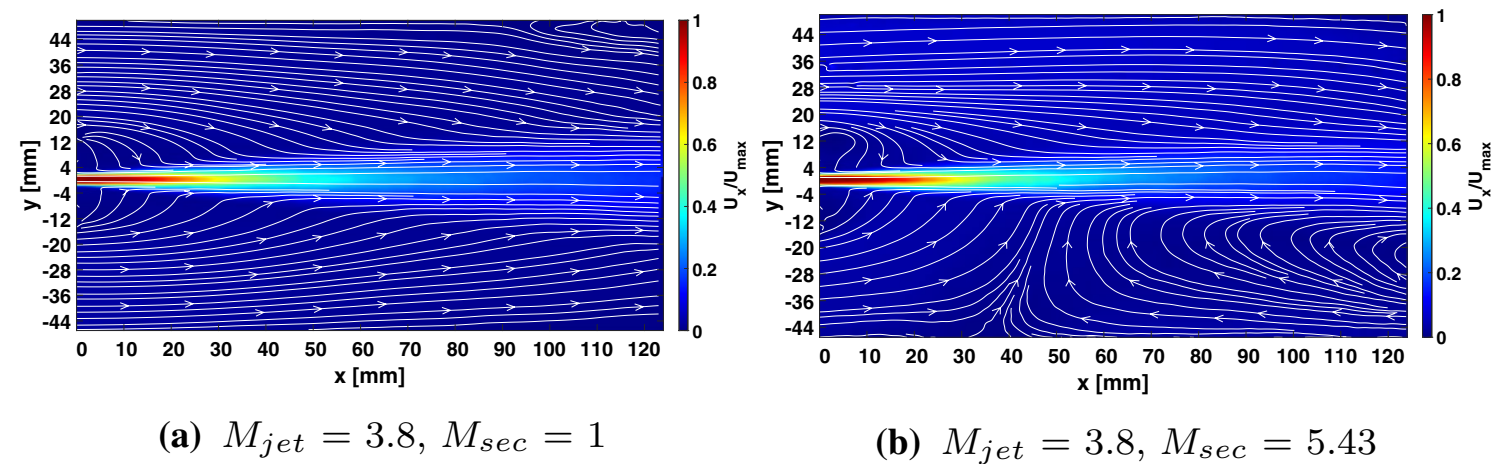

(b) $M_{\text {jet }}=3.8, M_{\text {sec }}=5.43$

Fig. 5 PIV results showing general flow features in the kiln vertical centerplane $z=0$. The coaxial jet is seen in the middle where red corresponds to high velocity and blue to low velocity. The white streamlines show the secondary co-flow and how it is entrained into the jet 

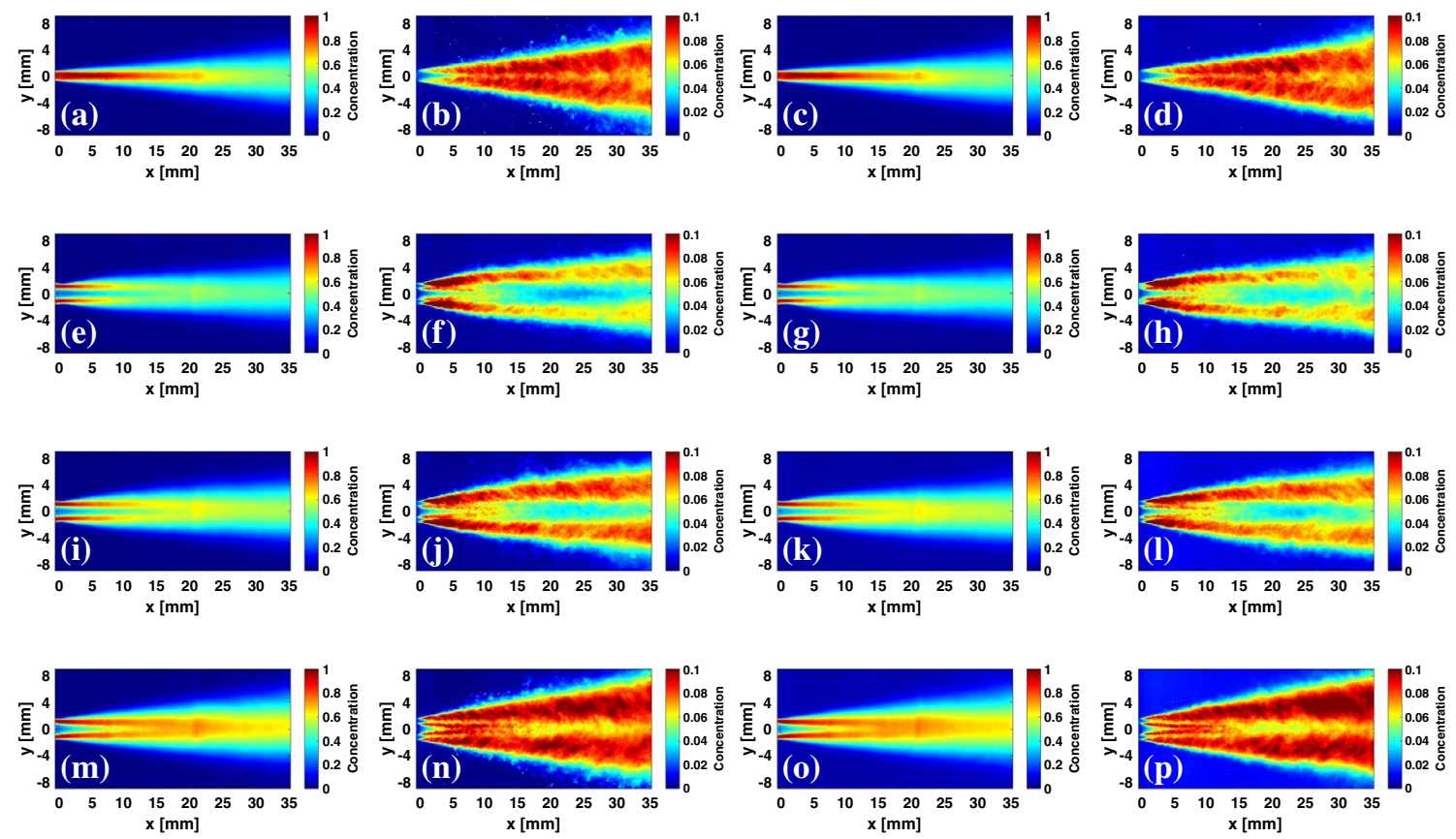

Fig. 6 Mean and rms fluctuations of concentration, close up of the part closest to the nozzle. $M_{\mathrm{sec}}=1$, the two columns to the left; $M_{\mathrm{sec}}=5.43$, the two columns to the right; $M_{\text {jet }}=0,0.5,1,3.8$ from top to bottom

observing the rms fluctuations (the second and fourth column in Fig. 6).

Note the region around $x=20 \mathrm{~mm}$ where the rms fluctuations decrease along the centerline for the coaxial cases. This region is accompanied in the mean image by an increase in concentration. This can be seen for all cases, including the single jet, and can be connected to the end of a wake that arises behind the back plate dividing the secondary flow streams (Larsson et al. 2012a).

In the wake region $(x<20 \mathrm{~mm})$, all jets develop without any major disturbances from the slow moving surrounding flow. After the end of the wake, around $x=20 \mathrm{~mm}$, the secondary flow more prominently affects the jet flow. In this region, also the jet centerline starts to experience the influence from the secondary flow. For the coaxial cases as $M_{\text {jet }}$ increases, the outer, coaxial stream dominates and penetrates the inner stream, resulting in higher concentration levels.

\subsection{Axial profiles}

The mean axial profiles of concentration and velocity downstream the jet entrance $(x=0)$ have similar shapes for the different secondary flow distributions (see Fig. 7). The major difference is the increased decay rate of the velocity along the centerline for the single jet $\left(M_{\text {jet }}=0\right.$, see Figs. $\left.7 \mathrm{c}, \mathrm{d}\right)$, which will be further discussed in the following section. For an uneven secondary flow distribution $\left(M_{\mathrm{sec}}=5.43\right)$, the jet centerline starts to deviate slightly toward the upper part of the confining kiln around $\mathrm{x}=80 \mathrm{~mm}$. This behavior is most apparent for $M_{\text {jet }}=3.8$. In general it is seen that the velocity decays faster than the concentration.

\subsection{Centerline values}

By studying the streamwise evolution of the centerline values, the axial decay rates can be determined. From these values, effects of the mixing between inner and outer coaxial jet and the surrounding fluid can be disclosed. Figure 8 shows the centerline decay of concentration and velocity. Since the PLIF seeding is done in different coaxial streams depending on if a single jet or a coaxial jet is studied, it is not possible to directly compare values between the cases. For a single jet, the seeding is done in the inner stream, and hence the concentration along the centerline will start at a high value and decrease further downstream as the jet mixes with the secondary flow. For a coaxial jet, the seeding is done in the outer stream to be able to distinguish between the inner and outer coaxial stream, as well as secondary flow. This means that the centerline concentration will start at a low value and initially increase as the inner coaxial stream mixes with the outer one. After the wake $(x \approx 20)$, the secondary flow starts to interact with the centerline, leading to a decrease of the concentration. Focus should therefore be on the rates of change (the derivative of the centerline in Fig. 8) instead of the actual values; see Figure 9. For easier comparison, the absolute values are shown. As can be seen in Fig. 8, no distinct difference in the rate of change can be seen between 


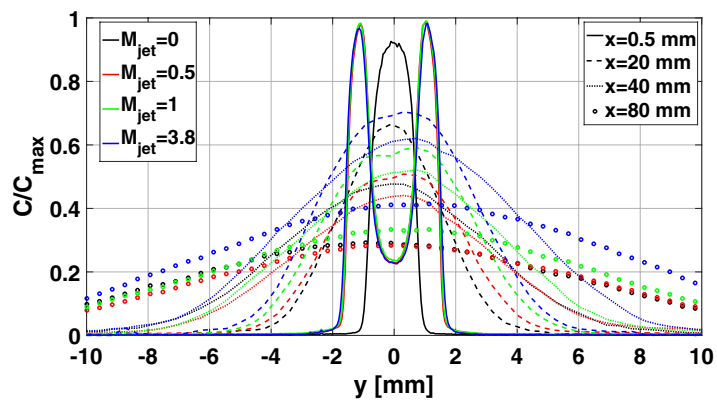

(a) $M_{s e c}=1$

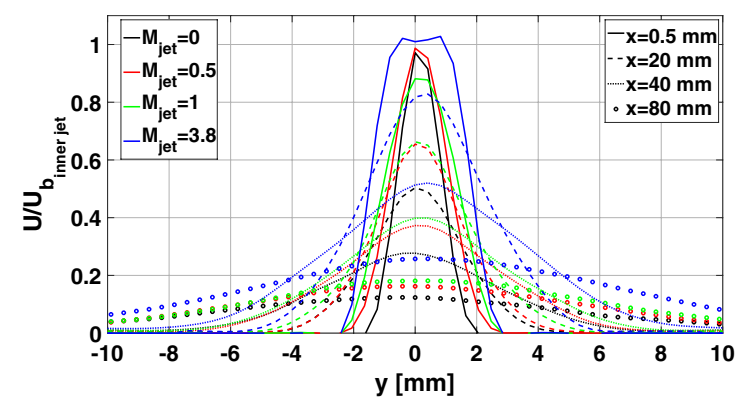

(c) $M_{\text {sec }}=1$

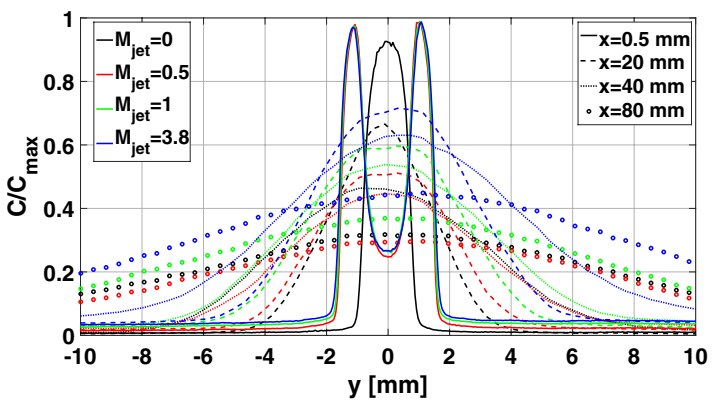

(b) $M_{\text {sec }}=5.43$

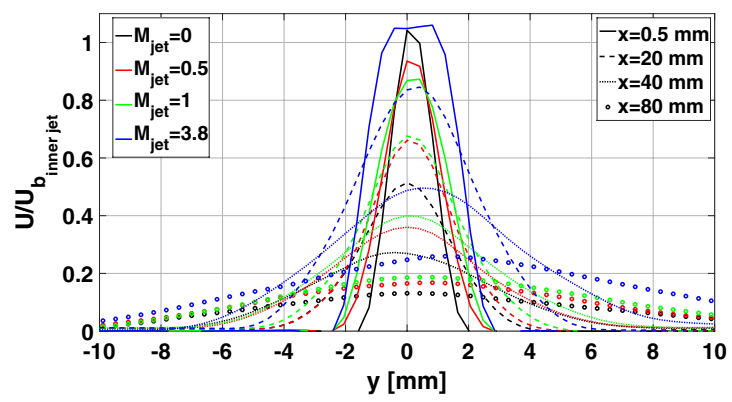

(d) $M_{\text {sec }}=5.43$

Fig. 7 Mean axial profiles of concentration $(\mathbf{a}, \mathbf{b})$, and velocity $(\mathbf{c}, \mathbf{d})$. The mean concentration is scaled with the maximum concentration. The mean axial velocity is scaled with the bulk velocity of the inner jet
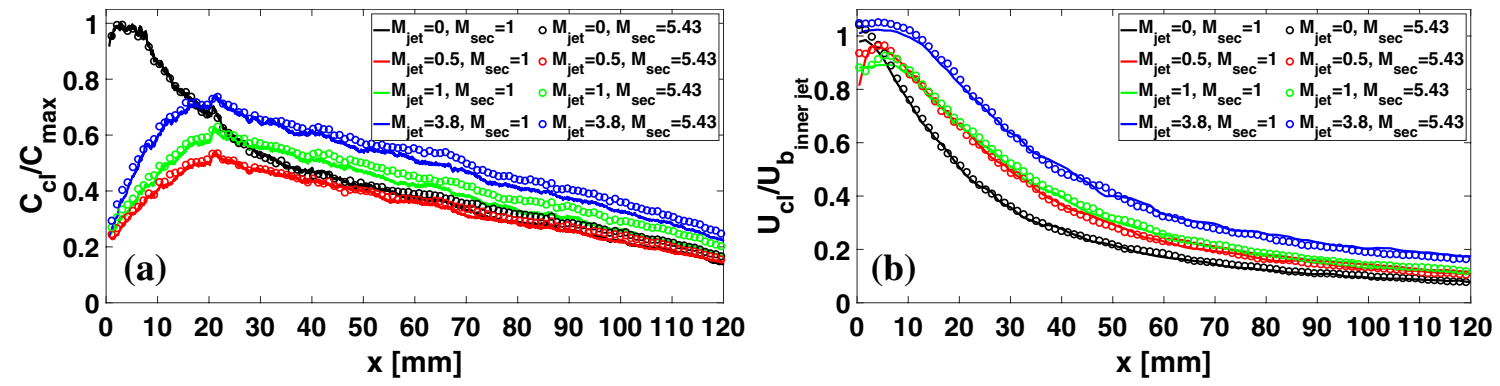

Fig. 8 Centerline decay of $\mathbf{a}$ concentration and $\mathbf{b}$ velocity. The mean centerline concentration is scaled with the maximum concentration. The mean axial centerline velocity is scaled with the bulk velocity of the inner jet

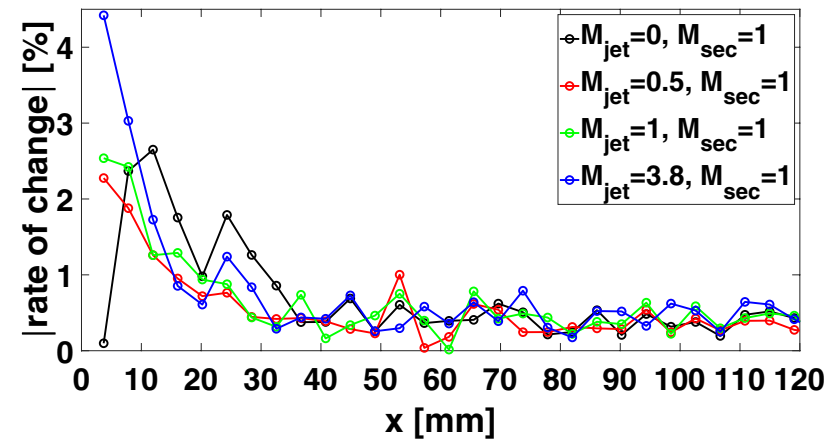

Fig. 9 Absolute value of rate of change of concentration (in percent). Each mark represents an average over $4 \mathrm{~mm}$
$M_{\mathrm{sec}}=1$ and $M_{\mathrm{sec}}=5.43$, hence only $M_{\mathrm{sec}}=1$ is shown in Fig. 9.

For the highest momentum ratio, $M_{\text {jet }}=3.8$, also the highest initial rate of change of the concentration can be found implying that the mixing along the centerline is larger for this case as compared to the other cases. For $M_{\text {jet }}>0$ and $x<20 \mathrm{~mm}$, the centerline values show the interaction between the inner and outer coaxial jet. Around $x=20 \mathrm{~mm}$, a local minimum can be seen for all cases, this is, as mentioned earlier, a result of the wake behind the back plate. After this point, the single jet, $M_{\text {jet }}>0$, initially has the highest rate of change, indicating more rapid mixing with the secondary flow as compared to the other cases in this region. After $x=40 \mathrm{~mm}$, the rates of change 
are more or less similar and steady, apart from a local minimum around $x=60 \mathrm{~mm} . M_{\text {jet }}=3.8$ though shows the highest value of the concentration (Fig. 8), indicating that the outer coaxial jet has mixed with the inner stream, but the secondary flow does not influence the centerline value substantially.

There is a slight difference between the secondary flow distributions when comparing the same momentum ratio, starting at approximately $x=40 \mathrm{~mm}$, see Fig. 8. This might be the result of the jet centerline deviating slightly from the geometrical kiln centerline for $M_{\mathrm{sec}}=5.43$. The rates of change are more or less the same (not explicitly shown here), but the values are higher for an uneven secondary flow distribution, $M_{\mathrm{sec}}=5.43$ (see Fig. 8).

The centerline velocity shows the same result regardless of secondary flow distribution with a faster decay of the single jet compared to a coaxial jet. This implies better mixing for this case. Again, it is seen that the velocity decays faster than the concentration. The difficulties associated with capturing the first nozzle diameters of the jet in the PIV-measurements are clearly visible in the velocity profile.

The fluctuations along the centerline can be seen for both concentration and velocity in Fig. 10. An increase in fluctuations (implying higher turbulence intensity) promotes the mixing and as can be seen in the concentration fluctuations (Fig. 10a), a peak corresponding to the mixing of the coaxial streams is found around $5-10 \mathrm{~mm}$ downstream the nozzle. The fluctuations of the coaxial cases then decrease until the end of the wake $(x \approx 20 \mathrm{~mm})$ when they again start to increase as the mixing with the secondary flow initiates. For the single jet, the fluctuations steadily increase. It is evident that the secondary flows' influence on the coaxial jet centerline is very limited for quite some distance downstream the nozzle due to the "protective" outer stream of the coaxial jet. The large variation of the velocity fluctuations close to the nozzle (Fig. 10b) again shows the difficulties in capturing the first

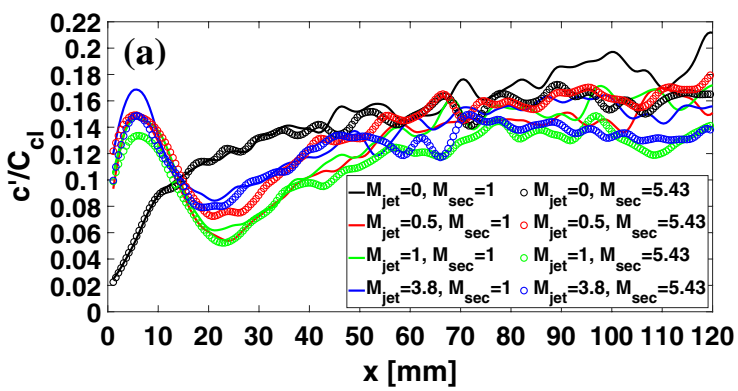

part of the jet in the PIV-measurements as compared to the PLIF-measurements.

\subsection{Reynolds flux and instantaneous flow fields}

Reynolds flux shows the turbulent transport of the scalar field, and can be seen in Fig. 11. To enable calculations of the Reynolds flux, the PLIF data was averaged and resampled on the same size grid as the PIV data. It should be noted that the measurements of the Reynolds fluxes show correct distribution, but the magnitude is underestimated due to the size of the interrogation area resulting in smoothing of the fluctuations. Four cases are shown, a single jet, $M_{\text {jet }}$ $=0$, and a coaxial jet, $M_{\text {jet }}=3.8$, for the two secondary flow distributions, $M_{\mathrm{sec}}=1$ and 5.43. When $M_{\text {jet }}$ increases, the turbulent flux of the passive scalar increases as well (note the different ranges of the colorbars). $M_{\text {jet }}=0$ though shows a faster transition toward a developed jet, well mixed with the secondary flow. The varying sign of the radial flux shows how the scalar is transported from the jet core to the secondary flow through the entrainment and mixing process. The axial turbulent flux clearly shows how the jet spreads and how the maximum scalar value decreases downstream. It is also obvious when comparing $M_{\mathrm{sec}}=1$ and 5.43 that the jet is drawn upward toward the dominant secondary flow for $M_{\text {sec }}=5.43$, especially when $M_{\text {jet }}=3.8$.

Instantaneous fields can be seen in Fig. 12 for the single jet, $M_{\text {jet }}=0$, and for a coaxial jet, $M_{\text {jet }}=3.8$ for the two secondary flow distributions $M_{\mathrm{sec}}=1$ and 5.43. The recirculation regions that develop in certain cases are clearly seen, and will be further discussed in the next section. The images show the turbulent nature and enhance the understanding of the flow dynamics.

\subsection{Spreading rates}

As described in the introduction, mixing relies on entrainment of fluid by large-scale structures in the flow. One way to visualize the entrainment rates is by plotting the inner

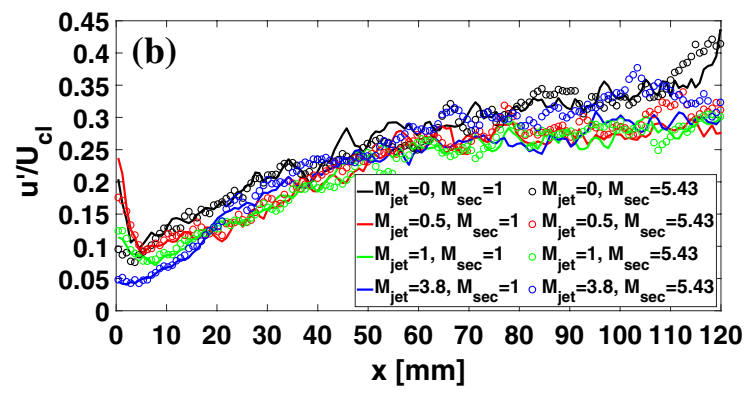

Fig. 10 Root mean square fluctuations along the jet centerline, a concentration and $\mathbf{b}$ velocity. The concentration fluctuations are scaled by the mean concentration along the centerline and the velocity fluctuations by the mean axial velocity along the centerline 


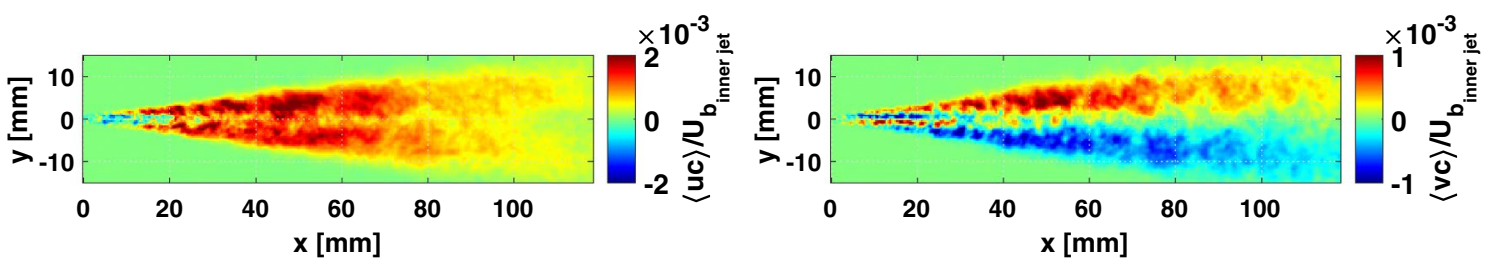

(a) $M_{j e t}=0, M_{\text {sec }}=1$

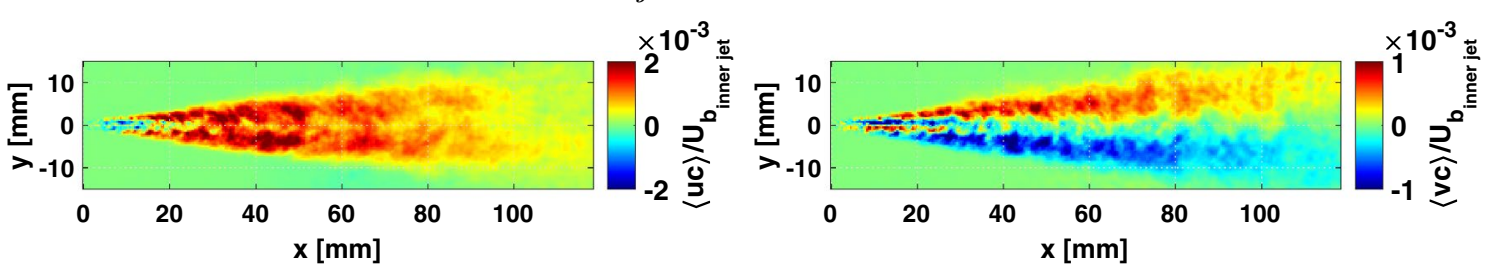

(b) $M_{j e t}=0, M_{\text {sec }}=5.43$

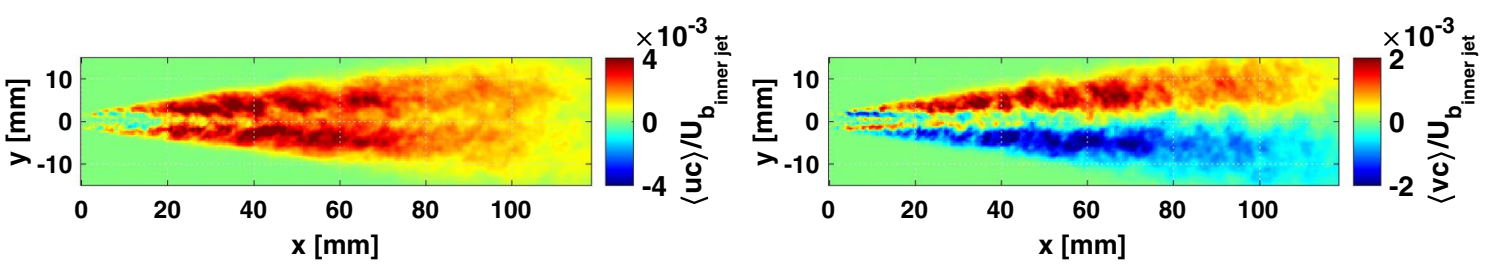

(c) $M_{\text {jet }}=3.8, M_{\text {sec }}=1$

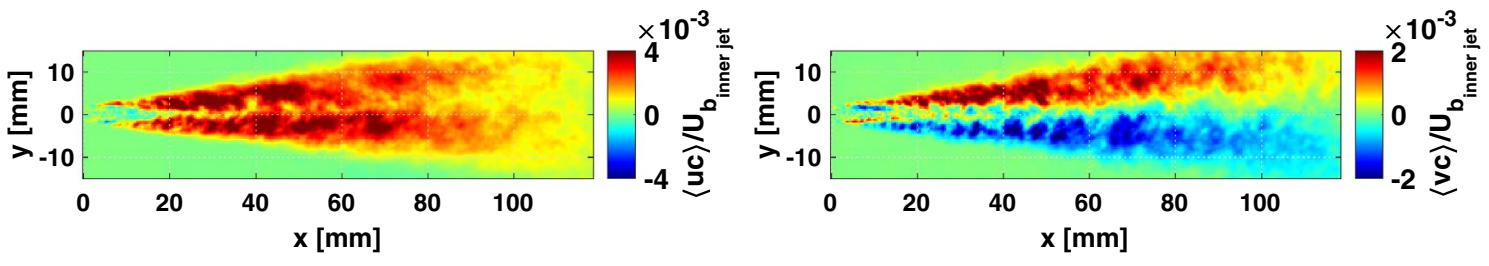

(d) $M_{\text {jet }}=3.8, M_{\text {sec }}=5.43$

Fig. 11 Reynolds flux, $\langle u c\rangle$ left column and $\langle v c\rangle$ right column. The flux is scaled by the bulk velocity of the inner jet

jet half-width which is the radial distance from the centerline $x$ axis where the velocity or concentration reaches half the centerline value for each streamwise position. Figure 13 shows the spreading of the jets and there is a clear difference between the two secondary flow distributions. When the distribution is uneven, $M_{\mathrm{sec}}=5.43$, the jet is slightly drawn upward toward the more dominating secondary flow. There is also a difference between the spreading of momentum and the passive scalar for these cases. Toward the end of the field of view, the spreading of the passive scalar below the jet increases sharply. This is connected to the recirculation zone that arises in the lower part of the kiln when the secondary flow distribution is uneven; see Fig. 12b, d. For $M_{\text {jet }}=3.8$ and even secondary flow distribution, $M_{\mathrm{sec}}=1$, the spreading rate of the concentration in the upper part of the jet deviates from the velocity with a sudden increase toward the end of the measurement region. This is connected to a developing recirculation zone above the jet that was clearly seen in instantaneous images; see Fig 12c. A trace of this recirculation zone can also be seen in the streamlines in the right upper corner of Fig. 5a. The jet momentum in this case is high enough to entrain all the secondary fluid, resulting in recirculation to overcome the excess momentum. This phenomenon does not occur when the secondary flow distribution is uneven. Hence, there is a clear, but not trivial interplay between $M_{\text {sec }}$ and $M_{\text {jet }}$ enabling a control of the jet development and therefore also the flame in the fullscale facility.

\subsection{Quantification of mixing}

Two measures of the mixing will be considered here, entrainment and probability density functions. The entrainment is based on mean data while the probability density functions are based on instantaneous data showing how the values fluctuate over time. 

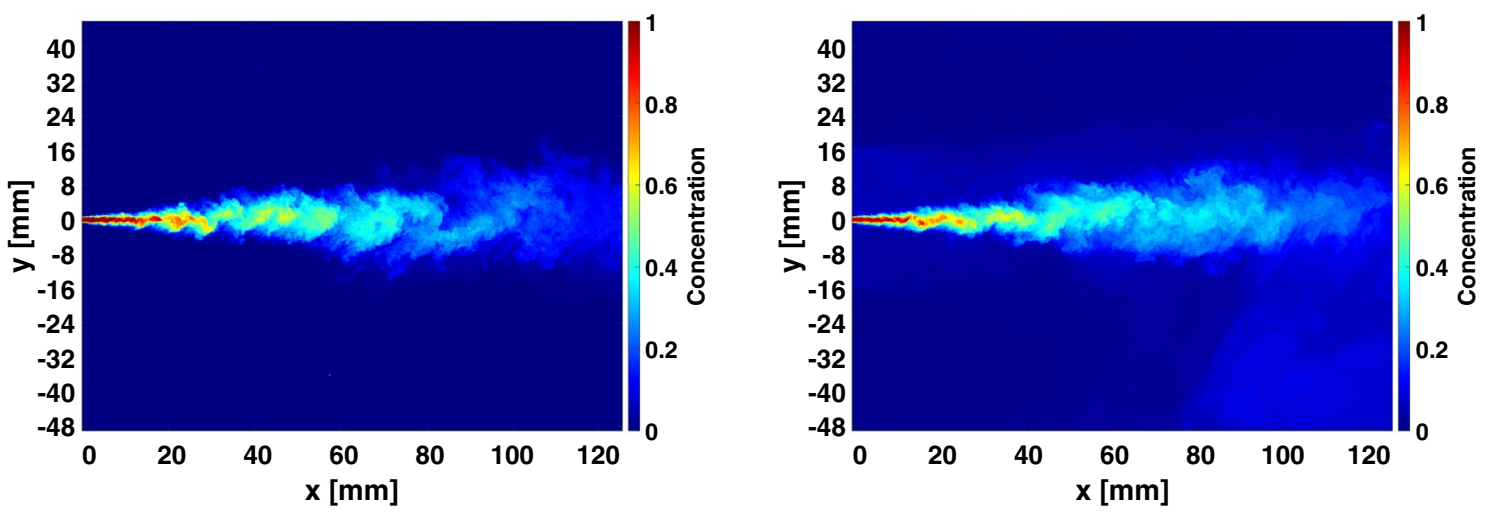

(a) $M_{j e t}=0, M_{s e c}=1$

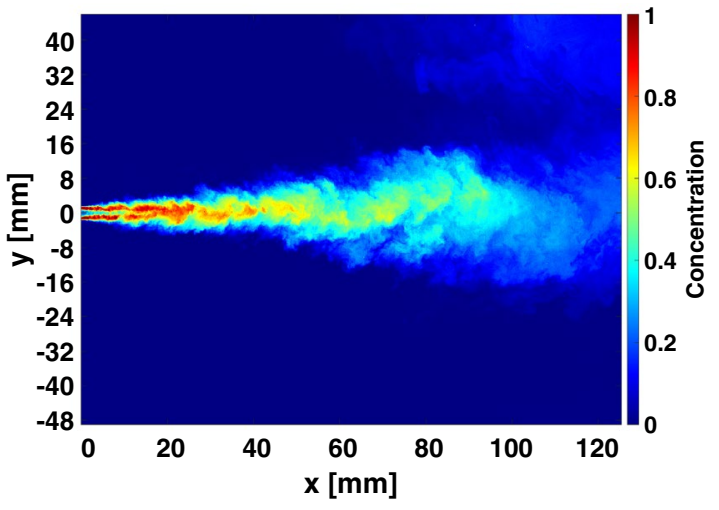

(c) $M_{\text {jet }}=3.8, M_{\text {sec }}=1$

(b) $M_{j e t}=0, M_{\text {sec }}=5.43$

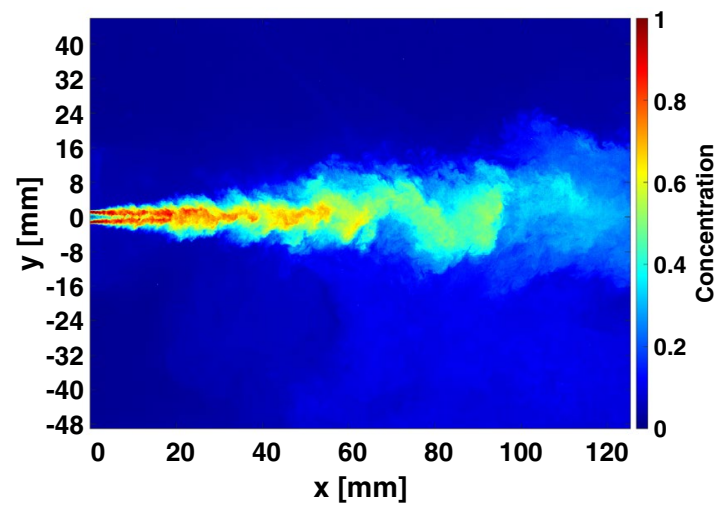

(d) $M_{j e t}=3.8, M_{s e c}=5.43$

Fig. 12 Instantaneous flow fields

\subsubsection{Entrainment}

One way to quantify the entrainment is to examine the increase in volumetric flow, $Q$, as the jet develops. This can be done by integrating the axial velocity profiles for each downstream position. The integration is performed over a cylindrical control surface with radius $r$, and hence it is assumed that the jet develops axisymmetrically. The radius is determined by the jet boundary and in this work it corresponds to the radial position where the axial velocity of the jet has reduced to $50 \%$ of the centerline velocity. The half-width was chosen since it provides a well-defined jet boundary, clearly distinguishable from the co-flowing secondary fluid. As can be seen in Fig. 13, the jet does not spread equally above and below the centerline, especially for the $M_{\mathrm{sec}}=5.43$ cases. In the calculation of the entrainment, the half-width used is the value above the centerline. This will result in a systematic error of the calculated volumetric flow that mainly influences the comparison between $M_{\mathrm{sec}}=$ 1 and $M_{\mathrm{sec}}=5.43$ for $x>50 \mathrm{~mm}$.

The volumetric flow is scaled by the total volumetric flow entering through the nozzle, $Q_{0}$, and this ratio gives

the entrainment coefficient. As can be seen in Fig. 14, the entrainment is highest for a single jet, $M_{\text {jet }}=0$, especially toward the end of the measurement region. Solely based on the entrainment coefficient, the conclusion would be that the single jet provides the largest mixing with the secondary flow.

\subsubsection{Probability density functions}

Until now, the focus has been on the time-averaged results, but the mean itself can be a poor representation of the actual fluid composition within the jet. This implies the need to differ between (molecularly) mixed fluid at a given composition and an average over mixed and unmixed fluids yielding the same composition in the mean (Dahm and Dimotakis 1987). Some indications regarding the mixing have also been based on the centerline decay. However, a faster axial decay could be the result of either more well mixed fluid or large-scale vortices engulfing outer, unmixed fluid and transporting it to the centerline. Hence, a faster axial decay is not a sufficient condition to verify mixing enhancement, and it is therefore necessary to investigate how a decrease in mean quantities 

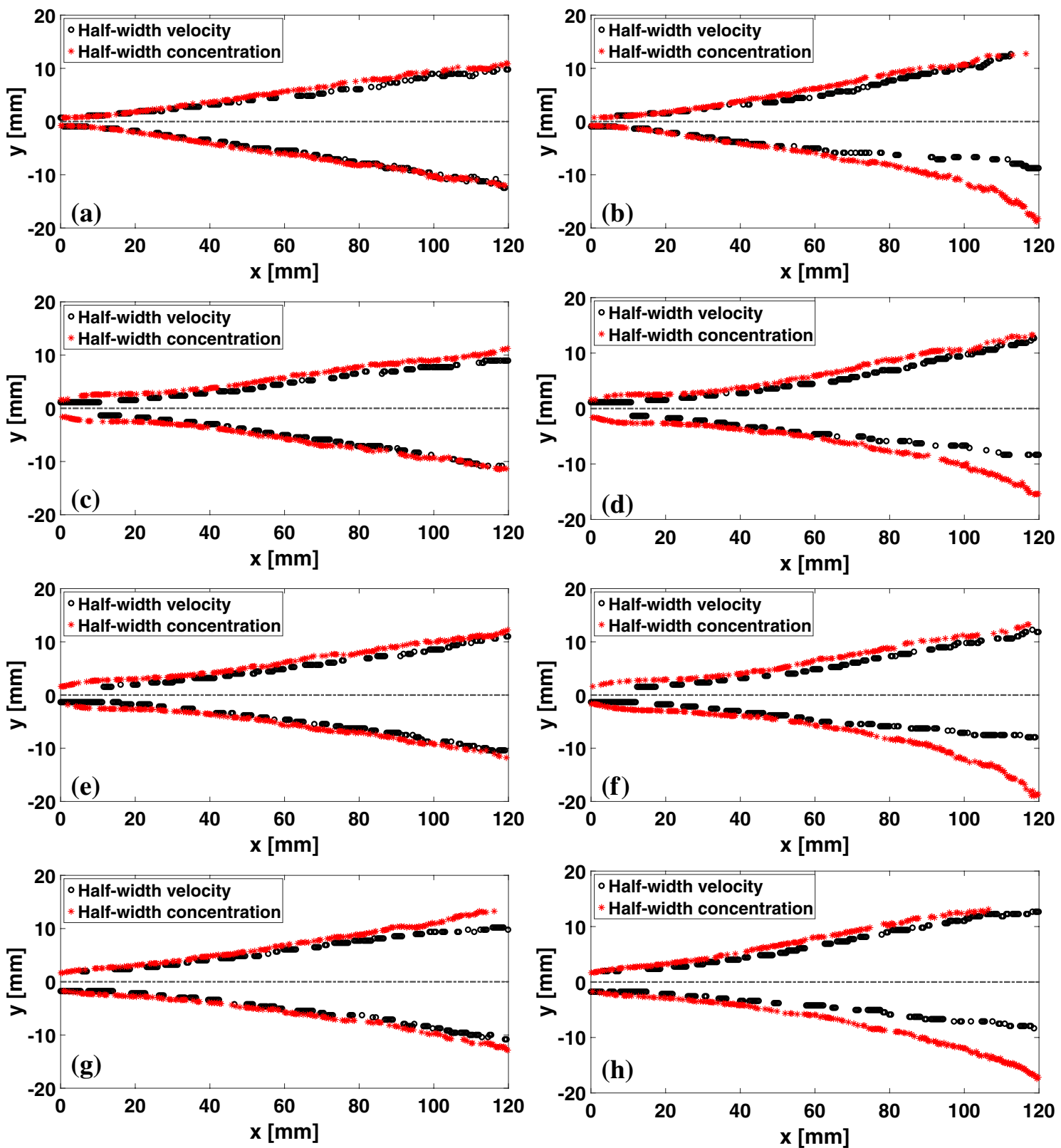

Fig. 13 Half-widths of velocity and concentration showing the spreading of momentum and the passive scalar. $M_{\text {sec }}=1$, left column; $M_{\text {sec }}=$ 5.43 , right column. $M_{\text {jet }}=0,0.5,1,3.8$ from top to bottom. The line in the middle shows the kiln centerline

is reflected in instantaneous values. This can for example be done through examining probability density functions (PDF) (Örlü and Alfredsson 2008; Mastorakos et al. 1996).

Therefore, to further investigate the mixing process, PDF:s of the concentration is plotted first in five positions along the centerline quite close to the jet nozzle; see Fig. 15. The instantaneous values are normalized with the corresponding mean centerline value and the values are captured during a period of $10 \mathrm{~s}$ implying 150 values for each position and case. The PDF:s along the centerline do not reveal any significant differences between the different secondary flow distributions, $M_{\mathrm{sec}}=1$ and $M_{\mathrm{sec}}=5.43$, in this region; hence, only $M_{\text {sec }}=1$ is shown for all different $M_{\text {jet }}$. Notice that the different colors of the PDF-curves are different positions along the $\mathrm{x}$-axis as indicated by the colored circles in the contour plots.

For a single jet, $M_{\text {jet }}=0$, it is seen that the maximum concentration is found a short distance downstream the nozzle. Only a few nozzle diameters downstream the PDF:s have become lower and wider indicating that mixing is taking place. For the coaxial cases, the seeding was done in the outer jet, hence the PDF closest to the nozzle starts at a low 


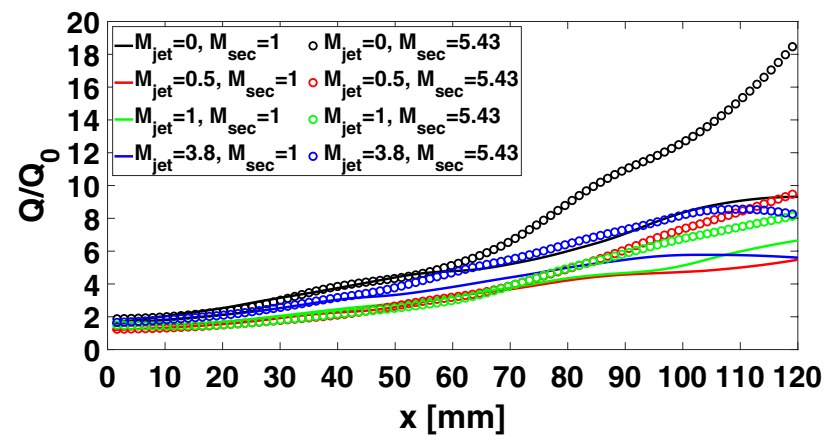

Fig. 14 Jet entrainment

value and increases, and the opposite from the single jet. $M_{\text {jet }}$ $=0.5$ shows a distinct difference compared to $M_{\text {jet }}=0$ in the shape of the PDF:s. Relatively high and narrow functions indicate that the concentration does not vary much in time. Note that the PDF:s first become wider when moving downstream as a result of the mixing between the inner and outer coaxial stream. Continuing further downstream, the peaks again become narrower and higher indicating less variation of the concentration in time. Around $x=20 \mathrm{~mm}$, the highest concentrations along the centerline can be found. This point coincides with the previously mentioned end of the wake behind the back plate and is defined by low concentration fluctuations (see Figs. 6 and 10) and a sudden increase of the mean value (see Fig. 8). $M_{\text {jet }}=1$ shows similar trends as $M_{\text {jet }}=0.5$. The PDF:s are though lower and wider in the wake, which implies better mixing between the inner and outer streams of the coaxial jet. They are also shifted toward higher concentration revealing that the outer coaxial jet influences the centerline more. The trends continue when increasing $M_{\text {jet }}$ to 3.8, the PDF:s are even lower, wider and shifted toward higher concentration. The second point (green color, $x=5 \mathrm{~mm}$ ) shows a PDF which is positively skewed with the tail toward higher concentration. This implies a probability of finding both low and high concentrations in this point. Comparing with the rms values in Figs. 6 and 10, it is seen that this point corresponds to high fluctuations due to the mixing of the inner and outer stream of the coaxial jet. An interesting observation is that the appearance of the PDF:s of $M_{\text {jet }}=3.8$ show closest resemblance to $M_{\text {jet }}=0$. The low and wide distributions for $M_{\text {jet }}=3.8$ though implies good mixing between inner and outer stream of the coaxial jet, but not with the secondary flow in the wake which is the case for $M_{\text {jet }}=0$. The results agree with the conclusion regarding the jet entrainment; the single jet provides the largest mixing with the secondary flow.

Additional PDF:s can be seen in Fig. 16 at three different axial downstream locations. For each downstream location (circular, triangular and square symbols), three radial positions are plotted (green, black and red) to follow the mixing process as the jet spreads. It should first be noted that again no distinct differences can be seen between $M_{\mathrm{sec}}=1$ and $M_{\text {sec }}=5.43$, apart from the red and green curves (circular markers) at $x \approx 20 \mathrm{~mm}$ for $M_{\text {jet }}=0$. Instantaneous values do not reveal any significant differences of the jet development when changing the secondary flow distribution around the coaxial jet, an interesting contradiction to the results from the analysis of the mean values (in particular Fig. 13). The reason for this is that the PDF analysis is done near
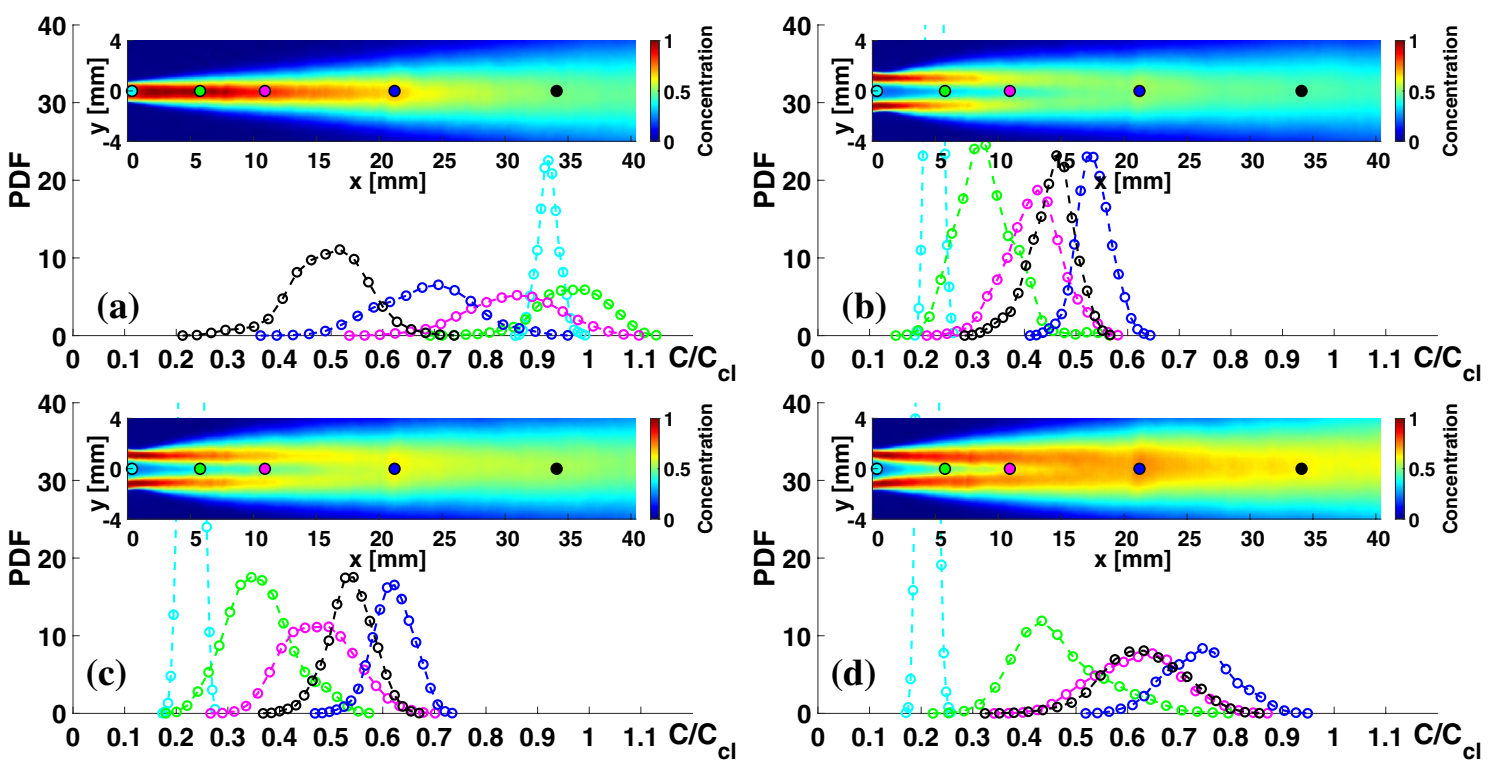

Fig. 15 Probability density functions along the centerline. $M_{\text {sec }}=1$ for all cases. a $M_{\text {jet }}=0, \mathbf{b} M_{\text {jet }}=0.5$, c $M_{\text {jet }}=1$, d $M_{\text {jet }}=3.8$. The different colors of the curves are different positions along the $\mathrm{x}$-axis as indicated by the colored circles in the plots 

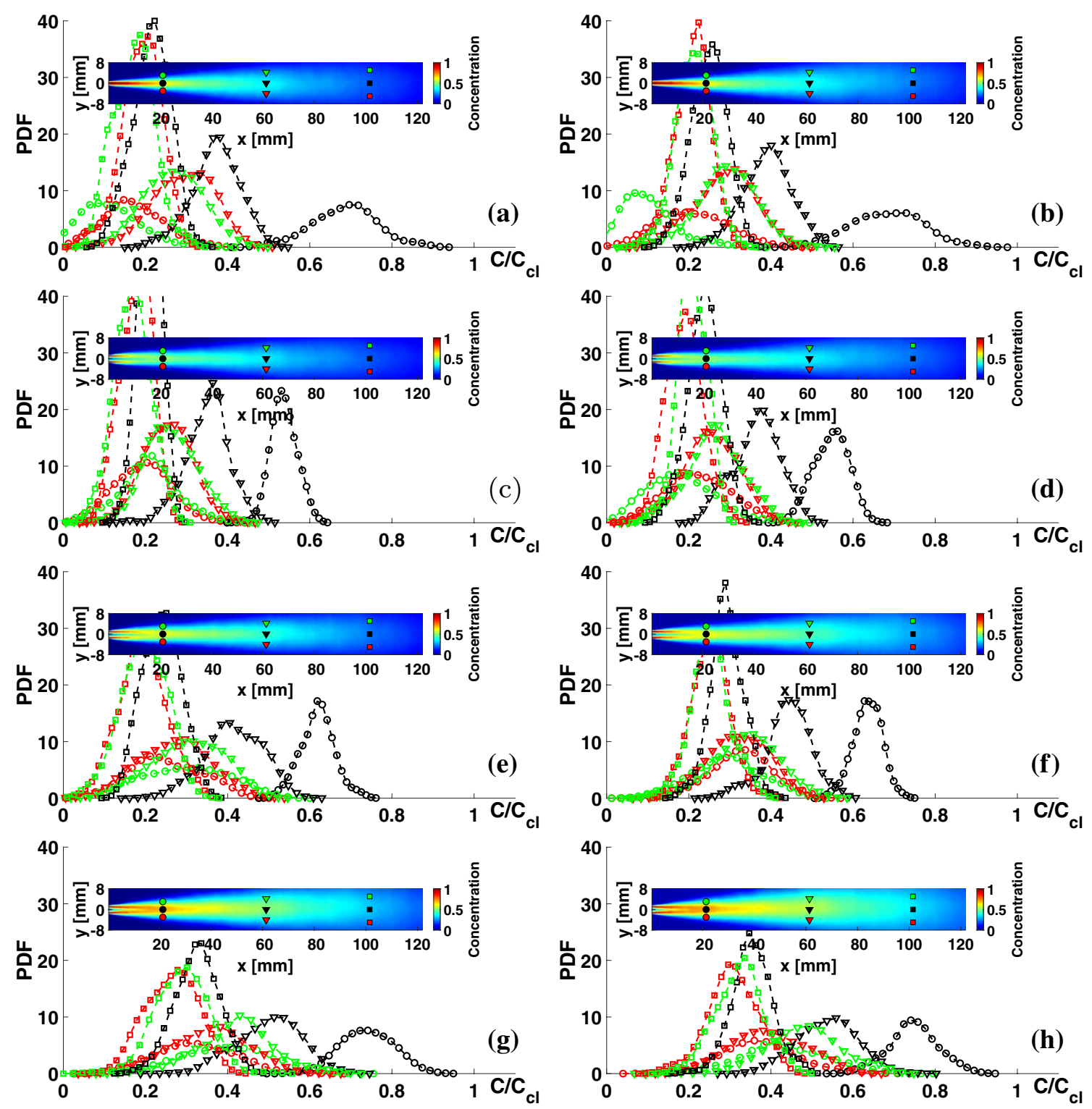

Fig. 16 Probability density functions, $M_{\text {sec }}=1$ left column, $M_{\text {sec }}=5.43$ right column. $M_{\text {jet }}=0,0.5,1,3.8$ from top to bottom. The symbols (circles, triangles and squares) denote position along the $x$ axis and the colors (green, black and red) radial position as indicated in the contour plots

the centerline. The secondary flow distribution affects the overall jet shape, but a variation in distribution cannot be observed close to the centerline. $M_{\text {jet }}=3.8$ shows the highest concentrations furthest downstream indicating a still significant jet (compare the square markers for all cases, $x \approx 100$ $\mathrm{mm}$ ), an effect of the larger total mass flow. Perfect (complete) mixing implies that each function in the radial direction collapses on the same, narrow curve. This cannot be seen for any case at the position furthest downstream (square markers, $x \approx 100 \mathrm{~mm}$ ), so mixing is still taking place. An interesting region is $x \approx 60 \mathrm{~mm}$. The point on the centerline (black triangle) shows PDF:s that are negatively skewed with a longer tail toward lower concentrations more or less distinguishable for all cases. This indicates that unmixed secondary fluid is entrained in vortical structures that reach the centerline here, which is also seen in the instantaneous images (Fig. 12). It is however not common enough to make a distinct impact on the mean value, though traces of it can be discerned in Figs. 8 and 9 for some cases.

The width of the PDF:s are in some sense connected to the size of the vortical structures within the flow. To achieve a wide variation of the concentration as a function of time, fluid from places far apart must pass the same spatial point during the time interval considered. This requires transport with large vortices. This information is completely missed when studying only the mean values. 


\section{Conclusions}

Confined, coaxial jets in a non-axisymmetric co-flow have been investigated. Both velocity and concentration were measured. The introduction of a coaxial stream affects the inner jet and initially decreases the mixing with the surrounding co-flow; the effect is enhanced as the momentum flow ratio of the coaxial jet increases.

Analysis of mean values resulted in the conclusion that the secondary flow distribution $\left(M_{\text {sec }}\right)$ affects the jet development. This was however not seen in the analysis of probability density functions of instantaneous concentration, showing the mixing process. Hence, the distribution of the secondary co-flow controls the shape and direction of the coaxial jet, but does not have a significant impact on the mixing process near the centerline.

Practical implications of this investigation are related to the possibility to better control a diffusion flame by introducing a coaxial stream. The main conclusion is that it is possible to affect the jet development and hence the flame length. The conclusion is based on the assumption that the outer, coaxial stream has a low mass flow, not enough to provide complete combustion, and hence the co-flowing, secondary fluid provides the air needed for the combustion process.

Future work includes further measurements to collect time-resolved data. By consistently seeding the rhodamine in the inner stream also for the coaxial cases, a better comparison between a single and coaxial jet would be facilitated. This approach will though be at the expense of the possibility to distinguish and quantify the mixing of all streams, inner and outer coaxial jet flow and co-flowing fluid. Tomographic, three-dimensional measurements can provide a deeper understanding of the jet development. Finally, by further varying the momentum flow ratio of the coaxial jet, $M_{\text {jet }}$, a more complete picture of the jet development and the mixing process can be achieved.

Acknowledgements This work was carried out within the VINNOVA STRIM-project "New digital 3D model of the Grate-Kiln pelletizing process for reduced energy usage and emissions" (2017-02170).

Funding Open access funding provided by Lulea University of Technology.

Open Access This article is licensed under a Creative Commons Attribution 4.0 International License, which permits use, sharing, adaptation, distribution and reproduction in any medium or format, as long as you give appropriate credit to the original author(s) and the source, provide a link to the Creative Commons licence, and indicate if changes were made. The images or other third party material in this article are included in the article's Creative Commons licence, unless indicated otherwise in a credit line to the material. If material is not included in the article's Creative Commons licence and your intended use is not permitted by statutory regulation or exceeds the permitted use, you will need to obtain permission directly from the copyright holder. To view a copy of this licence, visit http://creativecommons.org/licenses/by/4.0/.

\section{References}

Ahmed MR, Sharma SD (2000) Effect of velocity ratio on the turbulent mixing of confined, co-axial jets. Exp Therm Fluid Sci 22:19-33

Balarac G, Métais O (2005) The near field of coaxial jets: a numerical study. Phys Fluids 17(6):1-14

Coleman HW, Steele WG (1999) Experimentation and uncertainty analysis for engineers, 2nd edn. Wiley, New York

Dahm WJA, Dimotakis PE (1987) Measurements of entrainment and mixing in turbulent jets. AIAA J 25(9):1216-1223

Dahm W, Tryggvason G (1992) Vortex structure and dynamics in the near field of a coaxial jet. J Fluid Mech 241(32):371-402

da Silva C, Balarac G, Métais O (2003) Transition in high velocity ratio coaxial jets analysed from direct numerical simulations. J Turbul 4:18p

Favre-Marinet M, Schettini E (2001) Density field of coaxial jets with large velocity ratio and large density differences. Int J Heat Mass Transf 44(10):1913-1924

Favre-Marinet M, Camano EB, Sarboch J (1999) Near-field of coaxial jets with large density differences. Exp Fluids 26:97-106

Gibson M (1986) Hydrodynamics of confined coaxial jets. Encycl Fluid Mech 2:367-390

Han D, Mungal M (2001) Direct measurement of entrainment in reacting/nonreacting turbulent jets. Combust Flame 124(3):370-386

Larsson IAS, Granström BR, Lundström TS, Marjavaara BD (2012a) PIV analysis of merging flow in a simplified model of a rotary kiln. Exp Fluids 53:545-560

Larsson IAS, Lindmark EM, Lundström TS, Marjavaara BD, Töyrä S (2012b) Visualization of merging flow by usage of PIV and CFD with application to grate-kiln induration machines. J Appl Fluid Mech 5(4):81-89

Larsson IAS, Johansson SPA, Lundström TS, Marjavaara BD (2015a) PIV/PLIF experiments of jet mixing in a model of a rotary kiln. Exp Fluids 56:111

Larsson IAS, Lundström TS, Marjavaara BD (2015b) Calculation of kiln aerodynamics with two RANS turbulence models and by DDES. Flow Turbul Combust 94(4):859-878

Larsson IAS, Lundström TS, Marjavaara BD (2015c) The flow field in a virtual model of a rotary kiln as a function of inlet geometry and momentum flux ratio. ASME J Fluids Eng 137(10):101102

Larsson IAS, Lundström TS, Marjavaara BD (2016) Simulation of the flow field in an iron ore pelletizing kiln. Miner Metall Proces 33(3):144-148

Li W, Yuan M, Tong C, Carter C (2017) Experimental investigation of the effects of mean shear and scalar initial length scale on three-scalar mixing in turbulent coaxial jets. J Fluid Mech 817:183-216

Lotfiani A, Khalilarya S, Jafarmadar S (2013) A semi-analytical model for the prediction of the behavior of turbulent coaxial gaseous jets. Therm Sci 17(4):1221-1232

Mastorakos E, Shibasaki M, Hishida K (1996) Mixing enhancement in axisymmetric turbulent isothermal and buoyant jets. Exp Fluids 20(4):279-290

Melton LA, Lipp CW (2003) Criteria for quantitative plif experiments using high-power lasers. Exp Fluids 35:310-316

Mullinger P, Jenkins B (2008) Industrial and process furnaces: principles, design and operation, 1st edn. Butterworth-Heinemann, Oxford

Örlü R, Alfredsson PH (2008) An experimental study of the nearfield mixing characteristics of a swirling jet. Flow Turbul Combust $80(3): 323-350$ 
Parham J, Nathan G, Hill S, Mullinger P (2005) A modified thringnewby scaling criterion for confined, rapidly spreading, and unsteady jets. Combust Sci Technol 177:1421-1447

Pope SB (2000) Turbulent flows, 1st edn. Cambridge University Press, Cambridge

Rehab H, Villermaux E, Hopfinger EJ (1997) Flow regimes of largevelocity-ratio coaxial jets. J Fluid Mech 345:357-381

Schumaker S, Driscoll J (2012) Mixing properties of coaxial jets with large velocity ratios and large inverse density ratios. Phys Fluids 24(5):055101

Segalini A, Talamelli A (2011) Experimental analysis of dominant instabilities in coaxial jets. Phys Fluids 23(2):024103

Sreenivas K (2000) A semi-analytical model for the prediction of the behavior of turbulent coaxial gaseous jets. Phys Fluids 12(8):1221-1232
Villermaux E, Rehab H (2000) Mixing in coaxial jets. J Fluid Mech 425:161-185

Wieneke B (2015) PIV uncertainty quantification from correlation statistics. Meas Sci Technol 26(7):074002

Publisher's Note Springer Nature remains neutral with regard to jurisdictional claims in published maps and institutional affiliations. 